\title{
Different Dynamics and Entropy Rates in Quantum-Thermodynamics
}

\author{
A. Kato, M. Kaufmann, W. Muschik, D. Schirrmeister \\ Institut für Theoretische Physik, Technische Universität Berlin, Berlin, Germany \\ Communicated by K.H. Hoffmann, Chemnitz, Germany
}

Registration Number 838

\begin{abstract}
Different dynamics of the non-equilibrium canonical density operator, such as Canonical Dynamics, Linear Projection Dynamics, Generalized Robertson Dynamics, and Contact Time Dynamics, especially for time dependent work variables are derived. For two discrete systems in contact the rate of entropy is non-negative, if the contact time is short, and if one of the two discrete systems is in equilibrium and the compound system composed of both is isolated. The contact temperature is identified with one of the Lagrange parameters of the non-equilibrium canonical density operator of the compound system.
\end{abstract}

\section{Introduction}

As it is well known, quantum mechanics is a reversible theory [1]. Since quantum states of a system are described by its microscopic density operator $\varrho$, the fact of reversibility is expressed by an identical vanishing of the microscopical entropy rate in isolated systems

$$
\dot{S}^{0} \equiv 0, \quad S^{0}:=-k \operatorname{Tr}(\varrho \ln \varrho), \quad \operatorname{Tr} \varrho=1
$$

( $k$ is the Boltzmann constant).

An old question is how to obtain an irreversible description of quantum processes. The answer is manifold. One possibility is to introduce dissipative terms into Schrödinger's equation, or to postulate the existence of a microscopic entropy operator, both procedures are invented for generating an irreversible microscopic quantum theory. Another possibility of an irreversible description of systems is quantum-thermodynamics [2], a theory which is using restricted macroscopic information of the considered system, leaving its reversible microscopic background 
untouched. The information about the system is achieved by a so-called restricted set of relevant self-adjoint observables

$$
\begin{aligned}
& G_{j} \in \mathscr{B}:=\left\{G_{1}, G_{2}, \ldots, G_{n}\right\}, \quad\left(G_{1}, G_{2}, \ldots, G_{n}\right) \equiv \mathbf{G}, \\
& \text { with } G_{j}=G_{j}^{+} \quad \text { for all } j \in\{1,2, \ldots, n\} \text {. }
\end{aligned}
$$

According to [3] we will denote this restricted set of relevant observables $\mathscr{B}$ shortly as the beobachtungsebene (information level). Since the choice of this beobachtungsebene is equivalent to the choice of a state space in case of a macroscopic thermodynamical system, this irreversible quantum theory is called quantumthermodynamics, because also observables appear describing entropy, entropy production, and heat exchange.

In general the selfadjoint operators $\mathbf{G}$ of the beobachtungsebene depend on $m$ work variables a belonging to the system in consideration

$$
\mathbf{a}(t)=\left(a_{1}, a_{2}, \ldots, a_{m}\right)(t)
$$

which are time-dependent in thermodynamics. A special example for such a work variable is the system's volume $V(t)$ on which the Hamiltonian depends: $\mathscr{H}(V(t))$. In general we have $\mathbf{G}(\mathbf{a}(\mathrm{t}))$.

After having chosen the beobachtungsebene the microscopic density operator $\varrho(t)$ of the system is not determined by the expectation values of this restricted set of observables belonging to $\mathscr{B}$

$$
\mathbf{g}(t):=<\mathbf{G}(\mathbf{a}(t))>=\operatorname{Tr}[\varrho(t) \mathbf{G}(\mathbf{a}(t))]
$$

because there are other different microscopic density operators $\hat{\varrho}(t), \operatorname{Tr} \hat{\varrho}(t)=1$, satisfying also the $n$ relations (5)

$$
\mathbf{g}(t)=\operatorname{Tr}[\varrho(t) \mathbf{G}(\mathbf{a}(t))]=\operatorname{Tr}[\varrho(t) \mathbf{G}(\mathbf{a}(t))] .
$$

Consequently, the different density operators $\varrho(t)$ and $\varrho(t)$ are equivalent for all times with respect to $\mathscr{B}$. An operator $\hat{\varrho}$ for which (6) holds is called an accompanying density operator, because this concept is analogous to the definition of accompanying processes in non-equilibrium thermodynamics [4].

Since $\varrho$ is not determined by the set of the expectation values $\mathbf{g}$ we need an additional principle for choosing the density operator by which the system should be described in accordance with the restricted knowledge we have according to (5). The wellknown procedure stems from Jaynes $[5,6]$ : we choose that density operator $R$ which maximizes the (macroscopic) entropy of the system

$$
\begin{aligned}
& S:=-k \min _{\hat{\varrho}} \operatorname{Tr}(\varrho \varrho \ln \hat{\varrho})=-k \operatorname{Tr}(R \ln R), \quad \text { with } \\
& \mathbf{g}=\operatorname{Tr}(R \mathbf{G})=\operatorname{Tr}(\varrho \mathbf{G}), \quad \operatorname{Tr} R=1 .
\end{aligned}
$$


The maximization (7), that takes into account the constraints (6), yields to the wellknown result, that $R$ has the form of the generalized canonical operator [7]

$$
\begin{aligned}
& R=Z^{-1} \exp (-\boldsymbol{\lambda} \cdot \mathbf{G}), \quad Z=\operatorname{Tr} \exp (-\boldsymbol{\lambda} \cdot \mathbf{G}), \quad \text { or } \\
& R \equiv \exp \left(-\boldsymbol{\mu} \cdot \mathbf{G}^{\prime}\right), \quad \mathbf{G}^{\prime}=(\mathbf{1}, \mathbf{G}) \\
& \exp \left(-\mu_{0}\right)=Z^{-1}, \quad \mu_{j}=\lambda_{j}, j=1,2, \ldots, n .
\end{aligned}
$$

Here the $n$ quantities $\lambda$ have to be determined by the $n$ constraints (6).

An important remark is the following: the maximization (7) of the entropy can be executed at any instant, such that (9) is valid for all times

$$
R(t)=Z^{-1}(t) \exp [-\lambda(t) \cdot \mathbf{G}(\mathbf{a}(t))], \quad \operatorname{Tr} R(t)=1,
$$

or (9) is generated by a maximization only with respect to a fixed, but arbitrary time $t_{0}$. During the process $t_{0} \rightarrow t$ the initial density operator $R\left(t_{0}\right)$, which is of canonical form (9), changes its shape which may be different from (9). But during the process it remains an accompanying density operator $\hat{\varrho}(t)$ according to (6) which does not maximize the entropy $S(t)$ anymore. For these two cases the dynamics of the density operators are different. Consequences with respect to these different dynamics are investigated in the next sections.

The application of the maximum entropy principle does not imply that we restrict ourselves to equilibrium states, because the beobachtungsebene contains beyond the Hamiltonian and the particle number operator other operators not commuting with them in general. The generalized canonical operator $R$ therefore describes nonequilibrium states and is different from the grand canonical equilibrium density operator.

\section{Dynamics}

Time derivation of (7) yields to the entropy rate of the total system [8] at time $t_{0}$ of maximization

$$
\dot{S}\left(t_{0}\right)=-k \operatorname{Tr}\left[\dot{R}\left(t_{0}\right) \ln R\left(t_{0}\right)\right], \quad \text { and } \quad \operatorname{Tr} \dot{R}\left(t_{0}\right)=0
$$

according to $(8)_{2}$. Consequently in order to determine the entropy for all times we need the dynamics of the considered system represented by the time derivative $[\hat{\varrho}(t)]^{\bullet}$ of the accompanying process and its initial conditions. By maximization (7) at time $t>t_{0}$ we obtain the generalized canonical density operator $R(t)$ and the entropy $S(t)$ belonging to it

$$
\hat{\varrho}\left(t_{0}\right)=R\left(t_{0}\right), \quad \hat{\varrho}(t) \rightarrow R(t) \text { and } S(t) .
$$

There are now different possibilities of introducing $[\hat{\varrho}(t)]^{\bullet}$ which are investigated in the next sections. 


\subsection{Canonical dynamics}

The first possibility, called canonical dynamics, $C A$, requires that the accompanying process $\hat{\varrho}(t) \equiv R(t)$ has canonical form (9) for all times. Therefore $(13)_{1}$ yields for $t_{0} \equiv t$ by use of (12)

$$
\dot{S}_{C A}(t)=k \operatorname{Tr}[\lambda(t) \cdot \mathbf{G}(\mathbf{a}(t)) \dot{R}(t)] .
$$

$\dot{R}$ is determined by the time rates of the $\lambda$ appearing in (12) and by the given time rates of the work variables a on which the observables $\mathbf{G}$ in $\mathscr{B}$ depend. Consider for example a compound system consisting of two sub-systems separated by a movable piston. The Hamiltonians of the sub-systems clearly depend on the partial volumes which change in time, if the piston moves from a non-equilibrium position to an equilibrium one due to the different pressures in the sub-systems.

The independent rate variables are $\dot{\mathbf{a}}(t)$ and $\dot{\lambda}(t)$, or $\dot{\mathbf{a}}(t)$ and $\dot{\mathbf{g}}(t)$, if the $\dot{\lambda}$ are replaced by the $\dot{\mathbf{g}}$ by differentiating $(8)_{1}$. Consequently the time rate of $R$ is [8]:

$$
\dot{R}(t)=\frac{\partial R(t)}{\partial \mathbf{a}} \cdot \dot{\mathbf{a}}(t)+\frac{\partial R(t)}{\partial \lambda} \cdot \dot{\lambda}(t) .
$$

This type of dynamics is called canonical, if (12) is presupposed to be valid for all times, or more precisely.

Definition: A dynamics $R(t)$ is said to be canonical, if there exist time-dependent real parameters $\lambda(t)$ such that (12) holds for all time.

The coefficients $\partial R / \partial \mathbf{a}$ and $\partial R / \partial \lambda$ can be calculated by differentiation of (12), [8].

For demonstration we discuss the simple, but important example of two discrete systems $^{1)}$ in contact with each other. We consider the Hamiltonian of a closed (first) discrete system, denoted by $\square$, which is separated from its environment (the second discrete system), denoted by $*$, by a partition $\partial \square$. This partition determines the interaction between the considered discrete system and its environment. The timeindependent Hamiltonian of the isolated compound system is

$$
\begin{gathered}
\mathscr{H}=\mathscr{H}^{\square}(\mathbf{a})+\mathscr{H}^{*}(\mathbf{a})+\mathscr{H}^{\partial^{\square}}(\mathbf{a}), \\
{\left[\mathscr{H}^{\square}(t), \mathscr{H}^{*}(t)\right]=0, \quad \mathscr{H}^{\bullet}=0 .}
\end{gathered}
$$

Here $\mathscr{H}^{\square}(t)$ is the Hamiltonian of the discrete system in consideration, $\mathscr{H}^{*}(t)$ the Hamiltonian of its environment, and $\mathscr{H}^{\partial \square}(t)$ that of the interaction between the system and its environment, represented by the partition between them. Because the compound system is presupposed to be isolated, its energy is a constant

$$
E:=\operatorname{Tr}\left(\mathscr{H} R^{\text {dis }}\right)=\text { const } \rightarrow \operatorname{Tr}\left(\mathscr{H} \dot{R}^{\text {dis }}\right)=0 .
$$

Here the second equation follows from $(18)_{2} . R^{\text {dis }}$ is given by (21).

\footnotetext{
${ }^{1)}$ A discrete system, often also called lumped system, is always described by quantities belonging to the whole system. A field formulation of the system is not possible, not available, or not desirable.
} 
We now choose the beobachtungsebene of the isolated compound system in consideration

$$
\mathscr{B}^{\text {dis }}=\left\{\mathscr{H}, \mathscr{H}^{\square}(t), \mathscr{H}^{*}(t)\right\}
$$

to which for all times, according to (3) and (12), the following generalized canonical density operator belongs

$$
R^{d i s}=Z^{-1} \exp \left[-\left(\lambda^{\mathscr{H}} \mathscr{H}+\lambda^{\mathscr{H}^{\square}} \mathscr{H}^{\square}+\lambda^{\mathscr{H}^{*}} \mathscr{H}^{*}\right)\right] .
$$

According to (12) the $\lambda(t)$ depend on time

$$
\lambda^{\mathscr{H}}=\lambda^{\mathscr{H}}(t), \quad \lambda^{\mathscr{H}^{\square}}=\lambda^{\mathscr{H}^{\square}}(t), \quad \lambda^{\mathscr{H}^{*}}=\lambda^{\mathscr{H}^{*}}(t) .
$$

According to (15) the rate of entropy of the compound system is for all times by use of canonical dynamics

$$
\dot{S}_{C A}^{d i s}=k \operatorname{Tr}\left[\left(\lambda^{\mathscr{H}} \mathscr{H}+\lambda^{\mathscr{H}^{\square}} \mathscr{H}^{\square}+\lambda^{\mathscr{H}^{*}} \mathscr{H}^{*}\right) \dot{R}^{d i s}\right] .
$$

We now presuppose that the partition $\partial \square$ is inert [8], that means, the partition does not absorb or emit heat,

$$
0=\dot{E}^{\partial \square}:=\operatorname{Tr}\left(\mathscr{H}^{\partial \square} R^{\text {dis }}\right)^{\bullet}
$$

If we presuppose, that the partial Hamilton of the partition is independent of the work variables

$$
\dot{\mathscr{H}}^{\partial \square}=0
$$

we obtain from (24)

$$
\operatorname{Tr}\left(\mathscr{H}^{\partial \square} \dot{R}^{\text {dis }}\right)=0 .
$$

We now define the heat exchange (energy exchange at constant work variables) between $\square$ and $*$, [8] by

$$
\dot{Q}^{\square}:=\operatorname{Tr}\left(\mathscr{H}^{\square} \dot{R}^{\text {dis }}\right), \quad \dot{Q}^{*}:=\operatorname{Tr}\left(\mathscr{H}^{*} \dot{R}^{\text {dis }}\right)=-\dot{Q}^{\square} .
$$

Here the last equation follows from $(19)_{2}$, (18), and (26) by presupposing an inert partition. Introducing (26) into (23) we obtain the following entropy rate

$$
\frac{1}{k} \dot{S}_{C A}^{d i s}(t)=\left[\lambda^{\mathscr{H} \square}(t)-\lambda^{\mathscr{H}^{*}}(t)\right] \dot{Q}^{\square}(t), \quad \text { for } \dot{\mathbf{a}}(t) \neq \mathbf{0} \text { in general. }
$$

Thus for this non-equilibrium example of an isolated compound system the rate of entropy is proportional to the heat exchange between the sub-systems which in this 
case is the only source of entropy production. The interpretation of the factor $\left(\lambda^{\mathscr{H}^{\square}}-\lambda^{\mathscr{H}^{*}}\right)$ in (28) will be discussed in Section 3.

The expression (28) is the special entropy rate for a discrete system in contact with its environment. We now calculate the general entropy rate in canonical dynamics by inserting (16) into (15). We obtain (Appendix 1)

$$
\frac{1}{k} \dot{S}_{C A}=\lambda \cdot \mathscr{K} \cdot \dot{\lambda}-\lambda \cdot(\Delta \dot{\mathbf{G}} \mid \Delta \mathbf{G}) \cdot \lambda
$$

Here the following abbreviations were introduced: $\mathscr{K}$ is the symmetric, positive definite canonical correlation matrix

$$
\mathscr{K}:=(\Delta \mathbf{G} \mid \Delta \mathbf{G}), \quad \text { or } \quad \mathscr{K}_{i j}:=\left(\Delta G_{i} \mid \Delta G_{j}\right)
$$

and $(F \mid M)$ the generalized Mori product [9] by which the matrix elements of the canonical correlation matrix are defined

$$
(F \mid M):=\int_{0}^{1} \operatorname{Tr}\left(R F^{+} R^{u} M R^{-u}\right) d u .
$$

The symbol $\Delta$ introduces the deviation operator defined by

$$
\Delta X:=X-\operatorname{Tr}(R X) .
$$

The dependence of the entropy rate in canonical dynamics of the $\dot{\lambda}$ and of the $\dot{\mathbf{a}}$ is given by (29) for an unspecified beobachtungsebene of observables which depend on work variables. Its positivity will be discussed in Section 3.

\subsection{Linear projection dynamics}

Because $R$ can be represented by a projection of $\varrho[10]$ we are able to perform a different approach not using (16)

$$
R(t)=P(t)[\varrho(t)], \quad P^{2}(t)=P(t), \quad P(t) \text { linear on its domain. }
$$

Time differentiation yields

$$
\dot{R}(t)=(P(t)[\varrho(t)])^{\bullet}=\dot{P}(t)[\varrho(t)]+P(t)[\varrho(t)],
$$

and the dynamics of $R$ is determined by $\dot{P}$ and by $\dot{\varrho}$. If the microscopic dynamics is given by the von Neumann equation (reversible microscopic dynamics)

$$
\dot{\varrho}(t)=-i L \varrho(t)
$$

and $L$ is the Liouville operator defined by

$$
L \diamond:=(1 / \hbar)[\mathscr{H}, \diamond]
$$


we obtain from (34) the following dynamics (Appendix 2):

$$
\begin{aligned}
\dot{R}_{L P}(t)= & -i(P L+i \dot{P})(t) R(t) \\
& -\int_{t_{0}}^{t}(P L+i \dot{P})(t) T(t, s)(Q L-i \dot{P})(s) R(s) R(s) d s .
\end{aligned}
$$

Here $Q(t)$ and $T(t, s)$ are defined as follows:

$$
\begin{aligned}
& Q(t)[\diamond]:=1-P(t)[\diamond] \\
& \frac{\partial}{\partial s} T(t, s)=i T(t, s)(Q L-i \dot{P})(s), \quad \text { with the condition } \\
& T(t, t)=1 .
\end{aligned}
$$

Furthermore the initial preparation

$$
\varrho\left(t_{0}\right)=R\left(t_{0}\right)
$$

was used which is essential to get rid of the initial conditions in (37).

Definition: A dynamics (37) induced by $P(t)$ in (33) is called a linear projection dynamics.

Special cases of this dynamics are treated in the next sections.

Calculating the rate of entropy in linear projection dynamics according to (15) and (37) we obtain

$$
\begin{aligned}
\dot{S}_{L P}= & -i k \operatorname{Tr}(\boldsymbol{\lambda} \cdot \mathbf{G} P L R)+k \operatorname{Tr}(\boldsymbol{\lambda} \cdot \mathbf{G} \dot{P} R) \\
& -k \int_{t_{0}}^{t} \operatorname{Tr}((\boldsymbol{\lambda} \cdot \mathbf{G})(t)(P L+i \dot{P})(t) T(t, s)(Q L-i \dot{P})(s) R(s)) d s .
\end{aligned}
$$

\subsection{Robertson dynamics}

In linear projection dynamics $R(t)$ was represented by (33). There is another type of dynamics which connects the time derivatives of $R$ and $\varrho$ by a projector $P(t)$ instead of $(33)_{1}$ which is connecting the statistical operators themselves [8]

$$
\dot{R}(t)=P(t)[\dot{\varrho}(t)], \quad P^{2}(t)=P(t) .
$$

Definition: A type of dynamics induced by (43) is called a Robertson dynamics [12]. Often the projection operator in $(33)_{1}$ is especially identified with the so-called Kawasaki-Gunton operator [11]

$$
P(t)[\diamond] \equiv K(t)[\diamond]:=R(t) \operatorname{Tr}(\diamond)+\frac{\partial R}{\partial \mathbf{g}} \cdot(\operatorname{Tr}(\mathbf{G} \diamond)-\mathbf{g} \operatorname{Tr}(\diamond))
$$


which has according to (8) the following properties

$$
K(t)[\varrho]=R(t), \quad K(t)[R]=R(t)
$$

Hence we obtain for time-independent work variables

$$
\begin{aligned}
& \dot{\mathbf{a}} \equiv \mathbf{0}: \quad K(t)[\dot{\varrho}]=\dot{R}(t), \quad K(t)[\dot{R}]=\dot{R}(t), \\
& K(t)[K(t)[\diamond]]=K(t)[\diamond] .
\end{aligned}
$$

The projection properties for the time rates of the statistical operators (46) are only valid for time independent work variables. From this follows, that Robertson dynamics according to (44) can be performed only for work variables which are constant in time. In more detail we obtain from (44) by use of (8)

$$
K(t)[\dot{\varrho}]=\frac{\partial R}{\partial \mathbf{g}} \cdot \operatorname{Tr}(\mathbf{G} \varrho)=\frac{\partial R}{\partial \mathbf{g}} \cdot[\dot{\mathbf{g}}-\operatorname{Tr}(\dot{\mathbf{G}} \varrho)]
$$

Introducing canonical dynamics (16) this results in

$$
K(t)[\dot{\varrho}]=\dot{R}-\left[\frac{\partial R}{\partial \mathbf{a}}+\frac{\partial R}{\partial \mathbf{g}} \cdot \operatorname{Tr}\left(\frac{\partial \mathbf{G}}{\partial \mathbf{a}} \varrho\right)\right] \cdot \dot{\mathbf{a}}
$$

A comparison with (46) shows, that the use of Kawasaki-Gunton operator in Robertson dynamics presupposes work variables which are constant in time. In the next section we will look for dynamics which are consistent with time-dependent work variables, a standard situation in thermodynamics.

Equation (43) looks like (34), if formally $\dot{P}(t)$ is set to zero. Thus we obtain from (37) for Robertson dynamics

$$
\dot{R}_{R O}(t)=-i(P L)(t) R(t)-\int_{t_{0}}^{t}(P L)(t) T(t, s)(Q L)(s) R(s) d s
$$

with

$$
\frac{\partial}{\partial s} T^{0}(t, s)=i T^{0}(t, s)(Q L)(s)
$$

Replacing the projector $P(t)$ in (42) by the Kawasaki-Gunton operator (44) the entropy rate becomes (Appendix 3)

$$
\dot{S}_{L P}=-k \int_{t_{0}}^{t} \operatorname{Tr}((\lambda \cdot \mathbf{G})(t)(K L+i \dot{K})(t) T(t, s)(Q L-i \dot{K})(s) R(s)) d s
$$


According to (43) $\dot{K}$ has to be formally set to zero. Thus we obtain from (52) the rate of entropy in Robertson dynamics (43) by use of the Kawasaki-Gunton operator (44)

$$
\dot{S}_{R O}=-k \int_{t_{0}}^{t} \operatorname{Tr}\left((\boldsymbol{\lambda} \cdot \mathbf{G})(t)(K L)(t) T^{0}(t, s)(Q L)(s) R(s)\right) d s .
$$

\subsection{Generalized Robertson dynamics}

For including canonical dynamics into the formalism of projection dynamics we start out with a family of non-linear mappings $\mathscr{F}$ defined on normalized operators (we omit the family parameter a in the symbol $\mathscr{F}$ for the family of mappings), and we use the beobachtungsebene in the form of (10), [10]

$$
\mathscr{F}(X):=\exp \left[-\boldsymbol{\mu}(X, \mathbf{a}) \cdot \mathbf{G}^{\prime}(\mathbf{a})\right], \quad \operatorname{Tr} X=1 .
$$

Here the parameters $\boldsymbol{\mu}(X, \mathbf{a})$ are determined by the constraints (6)

$$
\operatorname{Tr}\left(\mathbf{G}^{\prime} X\right)=: \mathbf{g}(X, \mathbf{a}) \stackrel{!}{=} \operatorname{Tr}\left(\mathbf{G}^{\prime} \mathscr{F}(X)\right)=\operatorname{Tr}\left(\mathbf{G}^{\prime} \exp \left[-\boldsymbol{\mu}(X, \mathbf{a}) \cdot \mathbf{G}^{\prime}\right]\right)
$$

from which

$$
\boldsymbol{\mu}=\boldsymbol{\mu}(\mathbf{g}(X, \mathbf{a}), \mathbf{a})
$$

follows. According to (10) the identity $\mathbf{1}$ is enclosed in the extended beobachtungsebene $\left\{\mathbf{G}^{\prime}(\mathbf{a})\right\}$. Consequently we obtain from (55) and $(54)_{2}$

$$
\operatorname{Tr} \mathscr{F}(X)=1, \quad \operatorname{Tr}[\mathscr{F}(X)]^{\bullet}=0
$$

According to (55), $\mathscr{F}(\varrho)$ generates the expectation values of the observables which belong to the beobachtungsebene. Because $\mathscr{F}(X)$ has always canonical form according to (54), we have

$$
R(t)=\mathscr{F}(\varrho(t))
$$

instead of (33). Then (43) can be interpreted as the local mapping $P(X)$ of the family $\mathscr{F}$ in $(58)$

$$
[\mathscr{F}(X)]^{\bullet}=P(X)[\dot{X}] \rightarrow \dot{R}(t)=P(\varrho)[\grave{\varrho}(t)]
$$

connecting the time derivatives of $R$ and $\varrho$.

As it is proved in Appendix 4, the local mapping $P(\varrho)$ of $\mathscr{F}$ is for time dependent work variables

$$
P(\varrho)[\diamond]=\frac{\partial R}{\partial \mathbf{g}} \cdot \operatorname{Tr}\left(\mathbf{G}^{\prime} \diamond\right)+\frac{\partial R}{\partial \mathbf{g}} \cdot \operatorname{Tr}\left(\dot{\mathbf{G}}^{\prime} \varrho\right)+\frac{\partial R}{\partial \mathbf{a}} \cdot \dot{\mathbf{a}}
$$


with

$$
\begin{aligned}
\frac{\partial R}{\partial \mathbf{g}} & :=-R \int_{0}^{1} d \xi R^{-\xi} \mathbf{G}^{\prime}(\mathbf{a}) \cdot \frac{\partial \boldsymbol{\mu}}{\partial \mathbf{g}} R^{\xi} \\
\frac{\partial R}{\partial \mathbf{a}} & :=-R \int_{0}^{1} d \xi R^{-\xi}\left[\mathbf{G}^{\prime}(\mathbf{a}) \cdot \frac{\partial \boldsymbol{\mu}}{\partial \mathbf{a}}+\boldsymbol{\mu}(\mathbf{g}, \mathbf{a}) \cdot \frac{\partial \mathbf{G}^{\prime}}{\partial \mathbf{a}}\right] R^{\xi} .
\end{aligned}
$$

From (60) follows immediately

$$
P(\varrho)[\dot{\varrho}]=\dot{R}=\frac{\partial R}{\partial \mathbf{g}} \cdot \dot{\mathbf{g}}+\frac{\partial R}{\partial \mathbf{a}} \cdot \dot{\mathbf{a}}=P(R)[\dot{R}]
$$

which results in

$$
\operatorname{Tr} \dot{R}=0=\operatorname{Tr} \frac{\partial R}{\partial \mathbf{g}} \cdot \dot{\mathbf{g}}+\operatorname{Tr} \frac{\partial R}{\partial \mathbf{a}} \cdot \dot{\mathbf{a}} .
$$

Because $\partial R / \partial \mathbf{g}$ and $\partial R / \partial \mathbf{a}$ are independent of $\dot{\mathbf{g}}$ and $\dot{\mathbf{a}}$ which are also independent of each other, we obtain

$$
\operatorname{Tr} \frac{\partial R}{\partial \mathbf{g}}=\mathbf{0}, \quad \operatorname{Tr} \frac{\partial R}{\partial \mathbf{a}}=\mathbf{0} .
$$

Consequently, the traces of (61) and (62) results in

$$
\mathbf{g} \cdot \frac{\partial \boldsymbol{\mu}}{\partial \mathbf{g}}=\mathbf{0}, \quad \mathbf{g} \cdot \frac{\partial \boldsymbol{\mu}}{\partial \mathbf{a}}+\boldsymbol{\mu} \cdot \operatorname{Tr}\left(R \frac{\partial \mathbf{G}^{\prime}}{\partial \mathbf{a}}\right)=\mathbf{0} .
$$

Definition: The dynamics defined by $(59)_{2}$ and (60) is called Generalized Robertson Dynamics.

From (60) we are able to write down the generalized Kawasaki-Gunton operator which generalizes (44) to time dependent work variables

$$
\begin{aligned}
M(Y)[\diamond]:= & R(t) \operatorname{Tr}(\diamond)+\frac{\partial R}{\partial \mathbf{g}} \cdot\left[\operatorname{Tr}\left(\mathbf{G}^{\prime} \diamond\right)-\mathbf{g} \operatorname{Tr}(\diamond)\right] \\
& +\left[\frac{\partial R}{\partial \mathbf{g}} \cdot \operatorname{Tr}\left(\dot{\mathbf{G}}^{\prime} Y\right)+\frac{\partial R}{\partial \mathbf{a}} \cdot \dot{\mathbf{a}}\right][1-\operatorname{Tr}(\diamond)]
\end{aligned}
$$

The generalized Kawasaki-Gunton operator $M(\varrho)[\diamond]$ is different from the local mapping $P(\varrho)[\diamond]$ in $(60)$, but applied to $\varrho$ we obtain

$$
\begin{aligned}
& M(Y)[\varrho]=R, \quad M(Y)[R]=R, \quad \text { for all } Y, \\
& M(\varrho)[\dot{\varrho}]=\dot{R}, \quad M(R)[\dot{R}]=\dot{R}, \\
& M(Y)[M(Y)[\diamond]] \neq M(Y)[\diamond] .
\end{aligned}
$$

These equations are analogous to (45) and (46). Different from (47) we have (70). 
Although $(59)_{2}$ is also satisfied by $M(Y)[\diamond]$, the generalized Kawasaki-Gunton operator (67), as well as the original one in (44), is not a local mapping of $\mathscr{F}$ in (54). Because of (58) and $(54)_{1}$ canonical dynamics is a generalized Robertson dynamics.

As it is proved in Appendix 5, the generalized Robertson dynamics by use of $P(\varrho)$ in (60) is

$$
\begin{aligned}
\dot{R}_{G R}(t)= & P(\varrho)[-i L R](t)+\int_{t_{0}}^{t} d s P(\varrho)(t)[-i L T(t, s) W(s)] \\
& -\frac{\partial R}{\partial \mathbf{g}} \cdot \operatorname{Tr}\left\{\dot{\mathbf{G}}^{\prime} \int_{t_{0}}^{t} d s T(t, s) W(s)\right\}-\frac{\partial R}{\partial \mathbf{g}} \cdot \operatorname{Tr}\left(\dot{\mathbf{G}}^{\prime} R\right)-\frac{\partial R}{\partial \mathbf{a}} \cdot \dot{\mathbf{a}} .
\end{aligned}
$$

Here $W$ is defined by (see (126))

$$
W(s):=\frac{\partial R}{\partial \mathbf{g}} \cdot \operatorname{Tr}\left(\dot{\mathbf{G}}^{\prime} R\right)(s)+\frac{\partial R}{\partial \mathbf{a}} \cdot \dot{\mathbf{a}}(s)+Q(\varrho)[-i L R](s),
$$

and $T(t, s)$ satisfies the following differential equation (see (125))

$$
-\frac{\partial}{\partial s} T(t, s) \diamond=T(t, s)\left\{\frac{\partial R}{\partial \mathbf{g}} \cdot \operatorname{Tr}\left(\dot{\mathbf{G}}^{\prime} \diamond\right)(s)+Q(\varrho)[-i L \diamond](s)\right\} .
$$

We will not write down here the generalized Robertson dynamics induced by $M(Y)[\diamond]$. Also the entropy rates belonging to $P(\varrho)[\diamond]$ and $M(Y)[\diamond]$ will be woked out elsewhere.

\subsection{Contact time dynamics}

If the considered system is in contact with an environment, and if the contact time between them is sufficiently short (so that conduction problems are out of scope, and only the contact problem is considered), linear projection dynamics transforms into the special case of contact time dynamics [13]. In this context we define the following expressions

$$
\mathbf{v}:=-i \operatorname{Tr}(\mathbf{G} L R)
$$

which are called quantum-mechanical drift terms. If all these quantum-mechanical drift terms vanish for a chosen beobachtungsebene of the considered compound system (e.g. (20)), we obtain from linear projection dynamics (37) an approximation for small contact times $\Delta t:=t-t_{0}$ between the parts of the compound system (Appendix 6) by use of the Kawasaki-Gunton operator (44)

$$
\dot{R}=\dot{K} R-(K L+i \dot{K})(L-i \dot{K}) R \Delta t
$$

with the corresponding rate of entropy

$$
\dot{S}_{C T}=k \Delta t \operatorname{Tr}(\boldsymbol{\lambda} \cdot \mathbf{G}(-K L L R+K L i \dot{K} R-i \dot{K} L R-\dot{K} \dot{K} R)) .
$$


In the next section we will discuss the time rates of entropy in the four dynamics (29), (52), (53), and (76) especially with respect to their positive definiteness.

\section{Positive Rate of Entropy}

First of all we use a phenomenological argument to illustrate, that the rate of entropy is non-negative. For that purpose we recall: temperature is only defined for equilibrium states and therefore only for reversible processes [14]. Thus a redefinition of temperature with regard to non-equilibrium processes is necessary. This dynamical analogue of the thermostatic temperature is the so-called contact temperature [15, 16]. The defining inequality of the contact temperature $\Theta^{\square}$ of a closed discrete system whose partition $\partial \square$ is impervious to work and mass exchange, is as follows

$$
\lim _{t \rightarrow t_{0}+0}\left(\frac{1}{\Theta \square(t)}-\frac{1}{T^{*}\left(t_{0}\right)}\right) \dot{Q}^{\square}(t) \geq 0 .
$$

Here $T^{*}\left(t_{0}\right)$ is the thermostatic temperature of the system's environment which is supposed to be in equilibrium at time $t=t_{0}$, when the system gets in contact with its environment. The interpretation of the contact temperature is very easy: the defining inequality (77) determines the contact temperature $\Theta^{\square}\left(t_{0}\right)$ as that themostatic temperature of the system's environment, which causes, that at the contact time $t_{0}$ the net heat exchange between the system and its equilibrium environment vanishes.

Thus we find for arbitrary $t_{0}$

$$
\begin{aligned}
& \Theta^{\square}\left(t_{0}\right) \geq T^{*}\left(t_{0}\right) \rightarrow \dot{Q}^{\square}\left(t_{0}\right) \leq 0, \\
& \Theta^{\square}\left(t_{0}\right) \leq T^{*}\left(t_{0}\right) \rightarrow \dot{Q}^{\square}\left(t_{0}\right) \geq 0
\end{aligned}
$$

which is the statement of (77).

An interpretation of (28) with regard to the Second Law is now possible [17, 18]: if we identify

$$
\lambda^{\mathscr{H} \square}\left(t_{0}\right)+\Lambda(t) \equiv \frac{1}{k \Theta \square(t)}, \quad \lambda^{\mathscr{H}^{*}}(t)+\Lambda(t) \equiv \frac{1}{k T^{*}(t)},
$$

then from (28) and (77)

$$
\dot{S}_{C A}^{d i s} \geq 0
$$

follows by comparison. Therefore $\square$ represents a non-equilibrium system which is separated by an inert, diathermal, mass- and work-isolating partition $\partial \square$ from its surrounding environment $*$, the latter being always in equilibrium (reservoir). The meaning of $\Lambda(t)$ has been explained previously [8] and is of no interest here. The result which we obtain is the following: the entropy rate (28) is valid for a special, but sufficiently general beobachtungsebene (20). By use of canonical dynamics it is positive definite, if the heat exchange between parts of the compound system is 
interpreted by introducing the contact temperature which is defined by the inequality (77).

But the interesting question is just the other way round: what are the preassumptions to prove the positive definiteness of the entropy rate without use of contact temperature, and what is the quantum-thermodynamical interpretation of the contact temperature in that case?

Until now there is no way to prove the positivity of $\dot{S}_{C A}$ in (29), of $\dot{S}_{L P}(52)$, or of $\dot{S}_{R O}$ in (53). The conjecture is that these quantities are not definite unless additional preassumptions are made. One assumption to enforce positivity of (29) is to demand without any connection to microscopic dynamics a relaxation approach to equilibrium for the $\lambda$ in case of an isolated system which has constant work variables [8]

$$
\dot{\mathbf{G}} \equiv \mathbf{0}, \quad \dot{\boldsymbol{\lambda}}=-\alpha(\boldsymbol{\lambda}) \boldsymbol{\lambda}, \quad \alpha \geq 0
$$

Then (29) yields

$$
\dot{S}_{C A}=\alpha \lambda \cdot \mathscr{K} \cdot \lambda \geq 0
$$

because of the positivity of $\mathscr{K}$ and $\alpha$. But first of all there is no physical justification from the microscopic background for demanding the condition (82).

In case of contact time dynamics (75) and (76) we can prove the following

Proposition (Appendix 7): If all quantum-mechanical drift terms (74) vanish for the chosen beobachtungsebene, then we obtain by use of contact time dynamics for the entropy rate of the isolated compound system

$$
\begin{aligned}
& \dot{S}_{C T}=k(i \lambda \cdot L \mathbf{G}+\lambda \cdot \tilde{Q} \dot{\mathbf{G}} \mid i \lambda \cdot L \mathbf{G}+\lambda \cdot \tilde{Q} \dot{\mathbf{G}}) \Delta t \geq 0 \\
& \text { with } \tilde{Q}:=1-\tilde{P}, \quad \tilde{P}:=\mid \Delta \mathbf{G}) \cdot \mathscr{K}^{-1} \cdot(\Delta \mathbf{G} \mid .
\end{aligned}
$$

For deriving the positivity of the entropy production (81) the validity of the defining inequality (77) was pre-assumed. The proposition (84) now allows to derive an inequality which can be interpreted the other way round as the defining inequality (77). In case of constant work variables contact time dynamics (75) and the corresponding rate of entropy (84) yield

$$
\begin{aligned}
& \dot{\mathbf{a}} \equiv \mathbf{0} \rightarrow \dot{R}=-K L L R \Delta t, \\
& \dot{S}_{C T}=k(i \lambda \cdot L \mathbf{G} \mid i \lambda \cdot L \mathbf{G}) \Delta t \geq 0 .
\end{aligned}
$$

We now consider the discrete compound system described by the Hamiltonian (17) and by the beobachtungsebene (20). The partition between both subsystems is assumed to be inert (24). Then (87) yields

$$
\dot{S}_{C T}=k\left(\lambda^{\mathscr{H} \square} i L \mathscr{H}^{\square}+\lambda^{\mathscr{H}^{*}} i L \mathscr{H}^{*} \mid i \lambda \cdot L \mathbf{G}\right) \Delta t .
$$


The heat exchanges (27) are in contact time dynamics (Appendix 8)

$$
\dot{Q}^{\square}=\left(i L \mathscr{H}^{\square} \mid i \lambda \cdot L \mathbf{G}\right) \Delta t, \quad \dot{Q}^{*}=\left(i L \mathscr{H}^{*} \mid i \lambda \cdot L \mathbf{G}\right) \Delta t,
$$

so we can rediscover (28)

$$
\dot{S}_{C T}^{\text {dis }}=k\left(\lambda^{\mathscr{H}^{\square}}-\lambda^{\mathscr{H}^{*}}\right) \dot{Q}^{\square} \geq 0 .
$$

This inequality is now compared with the defining inequality of the contact temperature (77). After a short calculation we obtain the result

$$
1 / \Theta^{\square}=\alpha \lambda^{\mathscr{H}^{\square}}+c, \quad 1 / T^{*}=\alpha \lambda^{\mathscr{H}^{*}}+c,
$$

with constant $\alpha>0$ and $c$. A comparison with (80) demonstrates that relations

$$
\alpha=k \quad \text { and } \quad c=k \Lambda
$$

are valid. Therefore the connection between contact time dynamics and the defining inequality of contact temperature is evident.

\section{Discussion}

The choice of a state space for a thermodynamical discrete system is replaced in quantum-thermodynamics by introducing the beobachtungsebene, which is defined as a restricted set of relevant observables. By choosing the beobachtungsebene and by maximization of the system's entropy with respect to the constraints, the microscopic density operator is substituted by the generalized canonical (density) operator which exactly describes all properties of the system with respect to the chosen beobachtungsebene in non-equilibrium (Jaynes' procedure). As the construction of the generalized canonical operator is here local in time (it belongs e.g. to the initial time) the question arises, what dynamics the generalized canonical operator satisfies, especially if the work variables are time-dependent. Throughout the paper we are always investigating the influence of the time-dependence of the work variables, because this is the standard situation in thermodynamics.

Since the generalized canonical operator includes the work variables and the Lagrange parameters due to the maximization, its dynamics is determined by the time rates of the work variables and those of the Lagrange parameters. This kind of dynamics which preserves the form of the generalized canonical operator for all times is called canonical dynamics (Section 2.1).

Two other alternative types of dynamics are generated by projecting the microscopic density operator or by projecting its time rate to the accompanying process of maximal entropy

$$
R(t)=\mathscr{F}(\varrho(t)), \quad \dot{R}(t)=P(\varrho(t)) \dot{\varrho}(t) .
$$


Dynamics generated by $(93)_{1}$ are called Projection Dynamics and those generated by $(93)_{2}$ Generalized Robertson Dynamics (Section 2.4). In any case the dynamics of $R(t)$ is induced by the microscopic density operator $\varrho(t)$. If the mapping $\mathscr{F}$ is linear, we obtain Linear Projection Dynamics (Section 2.2) which is different from generalized Robertson dynamics according to a comparison of (34) with (93) ${ }_{2}$. Especially for short contact times linear projection dynamics transforms to Contact Time Dynamics (2.5). If the projector in generalized Robertson dynamics is linear, we obtain Robertson Dynamics (Section 2.3). Often the linear projection operator in Robertson dynamics is especially performed by the Kawasaki-Gunton operator which requires time-independent work variables. In contrast to that, the corresponding non-linear generalized Kawasaki-Gunton projector in generalized Robertson dynamics (67) takes into account time-dependent work variables. As it is well-known the generalized canonical operator $R(t)$ can be generated by a non-linear projection of the microscopic density operator $\varrho(t)$ (Section 2.4). Therefore canonical dynamics is a special case of generalized Robertson dynamics.

We derive the rates of entropy for canonical dynamics, linear projection dynamics and contact time dynamics, and finally for Robertson dynamics. The positivity of the entropy rate can be proved in following cases: in contact time dynamics in case of time-independent work variables and an unspecified beobachtungsebene of vanishing quantum-mechanical drift terms, and in case of a non-equilibrium system in contact with an equilibrium environment for which the contact temperature and heat exchange can be introduced for canonical and contact time dynamics. Here the macroscopic contact temperature can be interpreted by the Lagrange parameters of the generalized canonical operator, as well as the heat exchange between system and its equilibrium environment. Thus a quantum-thermodynamical foundation of contact temperature is possible.

Up to now the positivity of the entropy rate cannot be derived microscopically in full generality. In canonical dynamics in case of constant work variables we can enforce positivity of the entropy rate by a simple relaxation approach for the Lagrange parameters. But there is no microscopic proof, that the Lagrange parameters obey such a relaxation approach.

\section{Acknowledgement}

Financial support by the Deutsche Physikalische Gesellschaft (A.K.) and by the VISHAY-Company, D-95085 Selb, is gratefully acknowledged. We thank K.-H. Hoffmann (TU Chemnitz) for his advice to improve the paper.

\section{Appendices}

\subsection{Appendix 1}

The Kubo transformation of an observable $X$ is defined by

$$
\bar{X}:=\int_{0}^{1} R^{-u} X R^{u} d u
$$


According to [10] we obtain for the derivation of the generalized canonical operator (9) with respect to a parameter $y_{i}$

$$
\begin{aligned}
\frac{\partial R}{\partial y_{j}} & =-\frac{1}{Z^{2}} \frac{\partial Z}{\partial y_{j}} e^{-\lambda \cdot \mathbf{G}}+\frac{1}{Z} \frac{\partial}{\partial y_{j}} e^{-\lambda \cdot \mathbf{G}} \\
& =-\frac{1}{Z} \frac{\partial Z}{\partial y_{j}} R-\frac{1}{Z} \int_{0}^{1} e^{(\mu-1) \lambda \cdot \mathbf{G}} \frac{\partial(\boldsymbol{\lambda} \cdot \mathbf{G})}{\partial y_{j}} e^{-\mu \lambda \cdot \mathbf{G}} d \mu .
\end{aligned}
$$

Inserting

$$
\frac{\partial Z}{\partial y_{j}}=\operatorname{Tr}\left(\int_{0}^{1} e^{(\mu-1) \boldsymbol{\lambda} \cdot \mathbf{G}} \frac{\partial(-\boldsymbol{\lambda} \cdot \mathbf{G})}{\partial y_{j}} e^{-\mu \lambda \cdot \mathbf{G}} d \mu\right)=\operatorname{Tr}\left(e^{-\lambda \cdot \mathbf{G}} \frac{\partial(-\boldsymbol{\lambda} \cdot \mathbf{G})}{\partial y_{j}}\right)
$$

into (95) we get by use of (32), (94), [10]

$$
\frac{\partial R}{\partial y_{j}}=R \operatorname{Tr}\left(R \frac{\partial(\boldsymbol{\lambda} \cdot \mathbf{G})}{\partial y_{j}}\right)-R \int_{0}^{1} R^{-\mu} \frac{\partial(\boldsymbol{\lambda} \cdot \mathbf{G})}{\partial y_{j}} R^{\mu} d \mu=-R \overline{\left(\Delta \frac{\partial(\boldsymbol{\lambda} \cdot \mathbf{G})}{\partial y_{j}}\right)}
$$

Therefore we can transform (16) into

$$
\dot{R}=-R \overline{\Delta \mathbf{G}} \cdot \dot{\lambda}-R \lambda \cdot \overline{\Delta \dot{\mathbf{G}}}
$$

because $\lambda$ and $\mathbf{a}$ are independent of each other. By using the relation between the generalized Mori product (31) and the Kubo transformation (94)

$$
(X \mid Y)=\operatorname{Tr}\left(\overline{X^{+}} Y R\right)
$$

(98) and the definition (30), (15) results in

$$
\begin{aligned}
\dot{S}_{C A} & =-k \dot{\boldsymbol{\lambda}} \cdot(\Delta \mathbf{G} \mid \boldsymbol{\lambda} \cdot \mathbf{G})-k \boldsymbol{\lambda} \cdot(\Delta \dot{\mathbf{G}} \mid \boldsymbol{\lambda} \cdot \mathbf{G}) \\
& =-k \dot{\boldsymbol{\lambda}} \cdot \mathscr{K} \cdot \boldsymbol{\lambda}-k \boldsymbol{\lambda} \cdot(\Delta \dot{\mathbf{G}} \mid \Delta \mathbf{G}) \cdot \boldsymbol{\lambda} .
\end{aligned}
$$

\subsection{Appendix 2}

Starting out with the von Neumann equation (35), we write for (34):

$$
\dot{R}=-i P L \varrho+\dot{P} \varrho=-i(P L+i \dot{P}) R-i(P L+i \dot{P})(\varrho-R) .
$$


In order to get a differential equation for $R$ we have to remove $\varrho$ from this equation. Therefore we calculate the following expression

$$
\dot{\varrho}-\dot{R}=-i Q L \varrho-\dot{P} \varrho=-i(Q L-i \dot{P}) R-i(Q L-i \dot{P})(\varrho-R)
$$

which we multiply by $T(t, s)$ in (39) and integrate from the initial time $t_{0}$ to $t$ :

$$
\begin{aligned}
& \int_{t_{0}}^{t} T(t, s) \frac{d}{d s}(\varrho-R)(s) d s \\
& \quad=-i \int_{t_{0}}^{t} T(t, s)(Q L-i \dot{P})(s) R(s) d s-\int_{t_{0}}^{t} \frac{\partial}{\partial s} T(t, s)(\varrho-R)(s) d s .
\end{aligned}
$$

Considering the initial preparation (41) we obtain for the difference between the microscopic density operator and the generalized canonical operator

$$
(\varrho-R)(t)=-i \int_{t_{0}}^{t} T(t, s)(Q L-i \dot{P})(s) R(s) d s
$$

which we put in equation (101), so that we finally get the differential equation (37).

\subsection{Apendix 3}

Using the time derivative of the Kawasaki-Gunton operator (44)

$$
\dot{K}[\diamond]=\dot{R} \operatorname{Tr}(\diamond)+\left(\frac{\partial R}{\partial \mathbf{g}}\right)^{\bullet} \cdot(\operatorname{Tr}(\mathbf{G} \diamond)-\mathbf{g} \operatorname{Tr}(\diamond))+\frac{\partial R}{\partial \mathbf{g}} \cdot(\operatorname{Tr}(\dot{\mathbf{G}} \diamond)-\dot{\mathbf{g}} \operatorname{Tr}(\diamond))
$$

and (8) we obtain

$$
\dot{K} R=\dot{R}+\frac{\partial R}{\partial \mathbf{g}} \cdot \operatorname{Tr}(\dot{\mathbf{G}} R)-\frac{\partial R}{\partial \mathbf{g}} \cdot \dot{\mathbf{g}}=\dot{R}-\frac{\partial R}{\partial \mathbf{g}} \cdot \operatorname{Tr}(\mathbf{G} \dot{R}) .
$$

By taking $\operatorname{Tr}\left(\partial \diamond / \partial g_{k}\right)=\partial(\operatorname{Tr} \diamond) / \partial g_{k}$ into account

$$
\operatorname{Tr}\left(G_{j} \dot{K} R\right)=\operatorname{Tr}\left(G_{j} \dot{R}\right)-\operatorname{Tr}\left(\frac{\partial\left(G_{j} R\right)}{\partial g_{k}}\right) \operatorname{Tr}\left(G_{k} \dot{R}\right)=0,
$$

and

$$
\operatorname{Tr}\left(G_{j} K X\right)=\operatorname{Tr}\left(G_{j} R\right) \operatorname{Tr} X+\frac{\partial g_{j}}{\partial g_{k}} \operatorname{Tr}\left(G_{k} X\right)-\frac{\partial g_{j}}{\partial g_{k}} g_{k} \operatorname{Tr} X=\operatorname{Tr}\left(G_{j} X\right)
$$

are valid, and therefore the two first terms in (42) vanish, because of $[\boldsymbol{\lambda} \cdot \mathbf{G}, R]=0$, and we obtain (52). 


\subsection{Appendix 4}

If we replace in (55) $X$ by $\mathscr{F}(X)$, we obtain

$$
\operatorname{Tr}\left(\mathbf{G}^{\prime} \mathscr{F}(X)\right)=\mathbf{g}(\mathscr{F}(X), \mathbf{a})=\operatorname{Tr}\left(\mathbf{G}^{\prime} \mathscr{F}(\mathscr{F}(X))\right)=\mathbf{g}(X, \mathbf{a}) .
$$

Therefore $\boldsymbol{\mu}$ is invariant under this replacement

$$
\boldsymbol{\mu}(\mathbf{g}(X, \mathbf{a}), \mathbf{a})=\boldsymbol{\mu}(\mathbf{g}(\mathscr{F}(X), \mathbf{a}), \mathbf{a}),
$$

and $\mathscr{F}$ is a projector

$$
\mathscr{F}(\mathscr{F}(X))=\exp \left[-\boldsymbol{\mu}(\mathscr{F}(X), \mathbf{a}) \cdot \mathbf{G}^{\prime}\right]=\exp \left[-\boldsymbol{\mu}(X, \mathbf{a}) \cdot \mathbf{G}^{\prime}\right]=\mathscr{F}(X) .
$$

Further we have

$$
\begin{aligned}
{[\mathscr{F}(X)]^{\bullet} } & =\left\{\exp \left[-\boldsymbol{\mu}(X, \mathbf{a}) \cdot \mathbf{G}^{\prime}\right]\right\}^{\bullet} \\
& =-\mathscr{F}(X) \int_{0}^{1} d \xi[\mathscr{F}(X)]^{-\xi}\left[\boldsymbol{\mu}(X, \mathbf{a}) \cdot \mathbf{G}^{\prime}(\mathbf{a})\right]^{\bullet}[\mathscr{F}(X)]^{\xi}
\end{aligned}
$$

Performing the time derivative, taking (56) and (59), into account we obtain

$$
\begin{aligned}
P(X)[\diamond]= & -\mathscr{F}(X) \int_{0}^{1} d \xi[\mathscr{F}(X)]^{-\xi}\left\{\mathbf{G}^{\prime}(\mathbf{a}) \cdot \frac{\partial \boldsymbol{\mu}}{\partial \mathbf{g}} \cdot \operatorname{Tr}\left(\mathbf{G}^{\prime}(\mathbf{a}) \diamond\right)\right. \\
& \left.+\left[\mathbf{G}^{\prime}(\mathbf{a}) \cdot\left(\frac{\partial \boldsymbol{\mu}}{\partial \mathbf{g}} \cdot \operatorname{Tr}\left(\frac{\partial \mathbf{G}^{\prime}}{\partial \mathbf{a}} X\right)+\frac{\partial \boldsymbol{\mu}}{\partial \mathbf{a}}\right)+\boldsymbol{\mu}(X, \mathbf{a}) \cdot \frac{\partial \mathbf{G}^{\prime}}{\partial \mathbf{a}}\right] \cdot \dot{\mathbf{a}}\right\}[\mathscr{F}(X)]^{\xi}
\end{aligned}
$$

Replacing $X$ by $\varrho,(113)$ results in

$$
\begin{aligned}
P(\varrho)[\diamond]= & -R \int_{0}^{1} d \xi R^{-\xi}\left\{\mathbf{G}^{\prime}(\mathbf{a}) \cdot \frac{\partial \boldsymbol{\mu}}{\partial \mathbf{g}} \cdot \operatorname{Tr}\left(\mathbf{G}^{\prime}(\mathbf{a}) \diamond\right)\right. \\
& \left.+\left[\mathbf{G}^{\prime}(\mathbf{a}) \cdot\left(\frac{\partial \boldsymbol{\mu}}{\partial \mathbf{g}} \cdot \operatorname{Tr}\left(\frac{\partial \mathbf{G}^{\prime}}{\partial \mathbf{a}} \varrho\right)+\frac{\partial \boldsymbol{\mu}}{\partial \mathbf{a}}\right)+\boldsymbol{\mu} \cdot \frac{\partial \mathbf{G}^{\prime}}{\partial \mathbf{a}}\right] \cdot \dot{\mathbf{a}}\right\} R^{\xi}
\end{aligned}
$$

which satisfies $(59)_{2}$, by which we can read off (61), (62), and (60).

\subsection{Appendix 5}

From (60) we obtain

$$
\begin{aligned}
& P(\varrho)[\dot{\varrho}]-P(\varrho)[X]=\frac{\partial R}{\partial \mathbf{g}} \cdot \operatorname{Tr}\left(\mathbf{G}^{\prime}(\dot{\varrho}-X)\right), \\
& P(\varrho)[\dot{\varrho}-X]=\frac{\partial R}{\partial \mathbf{g}} \cdot \operatorname{Tr}\left(\mathbf{G}^{\prime}(\dot{\varrho}-X)\right)+\frac{\partial R}{\partial \mathbf{g}} \cdot \operatorname{Tr}\left(\dot{\mathbf{G}}^{\prime} \varrho\right)+\frac{\partial R}{\partial \mathbf{a}} \cdot \dot{\mathbf{a}}
\end{aligned}
$$


which results in

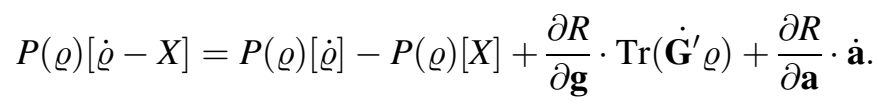

Consider

$$
\dot{R}=P(\varrho)[\varrho]=P(\varrho)[\varrho]-P(\varrho)[-i L R]+P(\varrho)[-i L R]
$$

From (117) we have

$$
\begin{aligned}
& P(\varrho)[-i L \varrho]-P(\varrho)[-i L R] \\
& \quad=P(\varrho)[-i L(\varrho-R)]-\frac{\partial R}{\partial \mathbf{g}} \cdot \operatorname{Tr}\left(\dot{\mathbf{G}}^{\prime} \varrho\right)-\frac{\partial R}{\partial \mathbf{a}} \cdot \dot{\mathbf{a}} \\
& \quad=P(\varrho)[-i L(\varrho-R)]-\frac{\partial R}{\partial \mathbf{g}} \cdot \operatorname{Tr}\left(\dot{\mathbf{G}}^{\prime}(\varrho-R)\right)-\frac{\partial R}{\partial \mathbf{g}} \cdot \operatorname{Tr}\left(\dot{\mathbf{G}}^{\prime} R\right)-\frac{\partial R}{\partial \mathbf{a}} \cdot \dot{\mathbf{a}} .
\end{aligned}
$$

Consequently we obtain

$$
\begin{aligned}
\dot{R}(t)= & P(\varrho)[-i L R]+P(\varrho)[-i L(\varrho-R)]-\frac{\partial R}{\partial \mathbf{g}} \cdot \operatorname{Tr}\left(\dot{\mathbf{G}}^{\prime}(\varrho-R)\right) \\
& -\frac{\partial R}{\partial \mathbf{g}} \cdot \operatorname{Tr}\left(\dot{\mathbf{G}}^{\prime} R\right)-\frac{\partial R}{\partial \mathbf{a}} \cdot \dot{\mathbf{a}} .
\end{aligned}
$$

Analogously to (117) we obtain for the projector (38)

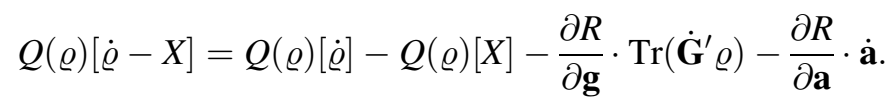

Consider

$$
\begin{aligned}
\dot{\varrho}-\dot{R} & =Q(\varrho)[\dot{\varrho}]-Q(\varrho)[-i L R]+Q(\varrho)[-i L R] \\
& =Q(\varrho)[\dot{\varrho}+i L R]+\frac{\partial R}{\partial \mathbf{g}} \cdot \operatorname{Tr}\left(\dot{\mathbf{G}}^{\prime} \varrho\right)+\frac{\partial R}{\partial \mathbf{a}} \cdot \dot{\mathbf{a}}+Q(\varrho)[-i L R]
\end{aligned}
$$

Here (121) was taken into account. Consequently (122) becomes

$$
\begin{aligned}
\dot{\varrho}-\dot{R}= & Q(\varrho)[-i L(\varrho-R)]+\frac{\partial R}{\partial \mathbf{g}} \cdot \operatorname{Tr}\left(\dot{\mathbf{G}}^{\prime}(\varrho-R)\right) \\
& +\frac{\partial R}{\partial \mathbf{g}} \cdot \operatorname{Tr}\left(\dot{\mathbf{G}}^{\prime} R\right)+\frac{\partial R}{\partial \mathbf{a}} \cdot \dot{\mathbf{a}}+Q(\varrho)[-i L R]
\end{aligned}
$$


Multiplication with $T(t, s)$ and integration yields

$$
\begin{array}{rl}
\int_{t_{0}}^{t} & d s T(t, s)(\dot{\varrho}-\dot{R})(s) \\
= & \int_{t_{0}}^{t} d s T(t, s)\left\{Q(\varrho)[-i L(\varrho-R)](s)+\frac{\partial R}{\partial \mathbf{g}} \cdot \operatorname{Tr}\left(\dot{\mathbf{G}}^{\prime}(\varrho-R)\right)(s)\right\} \\
& +\int_{t_{0}}^{t} d s T(t, s)\left\{\frac{\partial R}{\partial \mathbf{g}} \cdot \operatorname{Tr}\left(\dot{\mathbf{G}}^{\prime} R\right)(s)+\frac{\partial R}{\partial \mathbf{a}} \cdot \dot{\mathbf{a}}(s)+Q(\varrho)[-i L R](s)\right\} .
\end{array}
$$

We now demand that $T(t, s)$ satisfies the following differential equation

$$
\begin{gathered}
T(t, s)\left\{Q(\varrho)[-i L \diamond](s)+\frac{\partial R}{\partial \mathbf{g}} \cdot \operatorname{Tr}\left(\dot{\mathbf{G}}^{\prime} \diamond\right)(s)\right\}(\varrho-R)(s) \\
=-\frac{\partial}{\partial s} T(t, s)(\varrho-R)(s) .
\end{gathered}
$$

Introducing the abbreviation

$$
W(s):=\frac{\partial R}{\partial \mathbf{g}} \cdot \operatorname{Tr}\left(\dot{\mathbf{G}}^{\prime} R\right)(s)+\frac{\partial R}{\partial \mathbf{a}} \cdot \dot{\mathbf{a}}(s)+Q(\varrho)[-i L R](s)
$$

(124) results in

$$
\int_{t_{0}}^{t} d s\left\{T(t, s)(\dot{\varrho}-\dot{R})(s)+\frac{\partial}{\partial s} T(t, s)(\varrho-R)(s)\right\}=\int_{t_{0}}^{t} d s T(t, s) W(s)
$$

by taking (125) into account. Integration and taking (40) and (41) into consideration (127) results in

$$
(\varrho-R)(t)=\int_{t_{0}}^{t} d s T(t, s) W(s) .
$$

Inserting this into (120) we obtain (71). The operator $W(s)$ in (72) is defined by (126), and the differential equation (73) is given by (125).

\subsection{Appendix 6}

Since all quantum-mechanical drift terms (74) are pre-assumed to be vanishing, the Kawasaki-Gunton operator (44) yields the following equations:

$$
K L R=0 \quad \text { and } \quad Q L R=L R .
$$

We can now write (37) as

$$
\dot{R}=\dot{K} R-\int_{t_{0}}^{t}(K L+i \dot{K})(t) T(t, s)(L-i \dot{K})(s) R(s) d s
$$


As we consider short contact times $\Delta t:=t-t_{0}$, we make a Taylor expansion of the expression under the integral

$$
M(t, s):=(K L+i \dot{K})(t) T(t, s)(L-i \dot{K})(s) R(s)
$$

in $s$ and neglect quadratic and higher terms:

$$
\begin{aligned}
\int_{t_{0}}^{t} M(t, s) d s & =M(t, t) \int_{t_{0}}^{t} d s+\left.\frac{\partial M(t, s)}{\partial s}\right|_{s=t} \int_{t_{0}}^{t}(s-t) d s+\int_{t_{0}}^{t} o\left((s-t)^{2}\right) d s \\
& =M(t, t)\left(t-t_{0}\right)-\left.\frac{1}{2} \frac{\partial M(t, s)}{\partial s}\right|_{s=t}\left(t-t_{0}\right)^{2}+o\left(\left(t-t_{0}\right)^{3}\right) \\
& \approx M(t, t) \Delta t .
\end{aligned}
$$

Considering (40), we finally obtain (75).

\subsection{Appendix 7}

The expression for the rate of entropy (76) can be split into four terms (traces). DUe to (108) and (99) we can transform the first expression to

$$
\operatorname{Tr}(-(\boldsymbol{\lambda} \cdot \mathbf{G}) K L L R)=\operatorname{Tr}((L \lambda \cdot \mathbf{G})(L R))=(i L \lambda \cdot \mathbf{G} \mid i L \lambda \cdot \mathbf{G})
$$

Here the following identity is used in the last step [13]:

$$
L R=-R \overline{L \lambda \cdot \mathbf{G}},
$$

which can be derived from

$$
\begin{aligned}
{\left[\mathscr{H}, e^{-\lambda \cdot \mathbf{G}}\right] } & =\int_{0}^{1} \frac{d}{d \alpha}\left(e^{(1-\alpha)(-\lambda \cdot \mathbf{G})} \mathscr{H} e^{\alpha(-\lambda \cdot \mathbf{G})}\right) d \alpha \\
& =\int_{0}^{1} e^{(1-\alpha)(-\lambda \cdot \mathbf{G})}[\boldsymbol{\lambda} \cdot \mathbf{G}, \mathscr{H}] e^{\alpha(-\lambda \cdot \mathbf{G})} d \alpha .
\end{aligned}
$$

Due to (108) we also transform the second term into

$$
\operatorname{Tr}(\boldsymbol{\lambda} \cdot \mathbf{G} K L i \dot{K} R)=-\operatorname{Tr}((i L \lambda \cdot \mathbf{G}) \dot{K} R)
$$

Since the observables $\mathbf{G}$ only depend on time-dependent work variables a we can calculate (cf. (105))

$$
\dot{K} R=\frac{\partial K}{\partial t} R+\frac{\partial K}{\partial \mathbf{a}} R \cdot \dot{\mathbf{a}}=\frac{\partial R}{\partial \mathbf{a}} \cdot \dot{\mathbf{a}}-\frac{\partial R}{\partial \mathbf{g}} \cdot \operatorname{Tr}\left(\mathbf{G} \otimes \frac{\partial R}{\partial \mathbf{a}}\right) \cdot \dot{\mathbf{a}}
$$


Inserting (137) into (136), the second part vanishes because of the vanishing quantum-mechanical drift terms

$$
\operatorname{Tr}\left((i L \lambda \cdot \mathbf{G}) \frac{\partial R}{\partial g_{i}}\right)=-\lambda \cdot \frac{\partial}{\partial g_{i}} \operatorname{Tr}(i \mathbf{G} L R)=0,
$$

so we obtain by use of (97) and (99)

$$
\begin{aligned}
-\operatorname{Tr}((i L \lambda \cdot \mathbf{G}) \dot{K} R & =-\operatorname{Tr}\left((i L \lambda \cdot \mathbf{G}) \frac{\partial R}{\partial \mathbf{a}}\right) \cdot \dot{\mathbf{a}} \\
& =\left(\frac{\partial(\boldsymbol{\lambda} \cdot \mathbf{G})}{\partial \mathbf{a}} \cdot \dot{\mathbf{a}} \mid i L \boldsymbol{\lambda} \cdot \mathbf{G}\right)=(\boldsymbol{\lambda} \cdot \dot{\mathbf{G}} \mid i L \lambda \cdot \mathbf{G}) .
\end{aligned}
$$

In the last step (134) is used. According to (105), (134) and due to the vanishing quantum-mechanical drift terms the third term results in

$$
\begin{aligned}
-\operatorname{Tr}(\boldsymbol{\lambda} \cdot \mathbf{G} i \dot{K} L R) & =-\operatorname{Tr}\left((\boldsymbol{\lambda} \cdot \mathbf{G}) \frac{\partial R}{\partial \mathbf{g}}\right) \cdot \operatorname{Tr}(\dot{\mathbf{G}} i L R)=-\lambda \cdot \operatorname{Tr}(\dot{\mathbf{G}} i L R) \\
& =(i L \lambda \cdot \mathbf{G} \mid \boldsymbol{\lambda} \cdot \dot{\mathbf{G}})=(i L \lambda \cdot \mathbf{G} \mid \boldsymbol{\lambda} \cdot \tilde{Q} \dot{\mathbf{G}})
\end{aligned}
$$

if we use the definition (85). We see that the second and the third traces are identical. From (105), (107) and (137) we get

$$
\begin{aligned}
\dot{K}(\dot{K} R) & =\frac{\partial R}{\partial \mathbf{g}} \cdot \operatorname{Tr}(\dot{\mathbf{G}} \dot{K} R) \\
& =\frac{\partial R}{\partial \mathbf{g}} \cdot\left[\operatorname{Tr}\left(\dot{\mathbf{G}} \otimes \frac{\partial R}{\partial \mathbf{a}}\right)-\operatorname{Tr}\left(\dot{\mathbf{G}} \otimes \frac{\partial R}{\partial \mathbf{g}}\right) \cdot \operatorname{Tr}\left(\mathbf{G} \otimes \frac{\partial R}{\partial \mathbf{a}}\right)\right] \cdot \dot{\mathbf{a}}
\end{aligned}
$$

Inserting this expression into the fourth trace we obtain by use of (97) and (99)

$$
\begin{aligned}
& -\operatorname{Tr}((\boldsymbol{\lambda} \cdot \mathbf{G}) \dot{K} \dot{K} R) \\
& =\left(\Delta \frac{\partial(\boldsymbol{\lambda} \cdot \mathbf{G})}{\partial \mathbf{a}} \cdot \dot{\mathbf{a}} \mid \boldsymbol{\lambda} \cdot \dot{\mathbf{G}}\right)-\operatorname{Tr}\left((\boldsymbol{\lambda} \cdot \dot{\mathbf{G}}) \frac{\partial R}{\partial \mathbf{g}}\right) \cdot\left(\Delta \frac{\partial(\boldsymbol{\lambda} \cdot \mathbf{G})}{\partial \mathbf{a}} \cdot \dot{\mathbf{a}} \mid \mathbf{G}\right) .
\end{aligned}
$$

By using (97), (99), (3) and (30) we can show that

$$
\frac{\partial \mathbf{g}}{\partial \boldsymbol{\lambda}}=\operatorname{Tr}\left(\mathbf{G} \frac{\partial \mathrm{R}}{\partial \boldsymbol{\lambda}}\right)=-\operatorname{Tr}(\mathbf{G} R \overline{\Delta \mathbf{G}})=-(\Delta \mathbf{G} \mid \mathbf{G})=-\mathscr{K}
$$

By comparing (143) whith the following equality

$$
\underline{\underline{1}}=\operatorname{Tr}\left(\mathbf{G} \frac{\partial R}{\partial \mathbf{g}}\right)=\operatorname{Tr}\left(\mathbf{G} \frac{\partial R}{\partial \boldsymbol{\lambda}}\right) \cdot \frac{\partial \boldsymbol{\lambda}}{\partial \mathbf{g}}=\frac{\partial \mathbf{g}}{\partial \lambda} \cdot \frac{\partial \boldsymbol{\lambda}}{\partial \mathbf{g}}
$$


we get the identity

$$
\frac{\partial \boldsymbol{\lambda}}{\partial \mathbf{g}}=-\mathscr{K}^{-1}
$$

Now we can calculate the derivative of $R$ with respect to the expectation values by use of (145) and (97):

$$
\frac{\partial R}{\partial \mathbf{g}}=\frac{\partial R}{\partial \boldsymbol{\lambda}} \cdot \frac{\partial \boldsymbol{\lambda}}{\partial \mathbf{g}}=R \overline{\Delta \mathbf{G}} \mathscr{K}^{-1}
$$

From equations (146), (99) and the definition (85), (142) results in

$$
\begin{aligned}
-\operatorname{Tr}((\boldsymbol{\lambda} \cdot \mathbf{G}) \dot{K} \dot{K} R) & =\left(\boldsymbol{\lambda} \cdot \dot{\mathbf{G}} \mid \tilde{Q} \Delta \frac{\partial(\boldsymbol{\lambda} \cdot \mathbf{G})}{\partial \mathbf{a}} \cdot \dot{\mathbf{a}}\right)=(\boldsymbol{\lambda} \cdot \tilde{Q} \dot{\mathbf{G}} \mid \boldsymbol{\lambda} \cdot \tilde{Q} \dot{\mathbf{G}}), \\
\text { because } \mid \tilde{Q} \Delta \mathbf{G}) & =0 \quad \text { is valid. }
\end{aligned}
$$

After adding up the four terms (133), (139), (140) and (147) we obtain (84).

\subsection{Appendix 8}

If the work variables are constant in contact time dynamics, we can use (86) to calculate

$$
\operatorname{Tr}\left(G_{j} \dot{R}\right)=\operatorname{Tr}\left(\left(L G_{j}\right)(L R)\right) \Delta t
$$

by taking (108) into account. From (134) we obtain (89).

\section{References}

[1] Dougherty, J. P., Foundations of non-equilibrium statistical mechanics, Phil. Trans. R. Soc. Lond. A, 346 (1994), 259-305.

[2] Muschik, W., Kaufmann, M., Statistical Physics and Thermodynamics of Nonlinear Nonequilibrium Systems -Statistical Physics 18 Satellite Meeting, Ed. Ebeling, W., Muschik, W., World Scientific, Singapore, 1993, 229-242.

[3] Schwegler, H., Verallgemeinerte physikalische Entropien auf informationstheoretischer Basis, Z. Naturforsch., 20a (1965), 1543-1553.

[4] Muschik, W., Non-Equilibrium Thermodynamics with Application to Solids, in: Ed. Muschik, W., Fundamentals in Nonequilibrium Thermodynamics, Springer, Wien, 1993.

[5] Jaynes, E. T., Information theory and statistical mechanics I, Phys. Rev., 106 (1957), 620 630.

[6] Jaynes, E. T., Information theory and statistical mechanics II, Phys. Rev., 108 (1957), 171190.

[7] Jaynes, E. T., The Maximum Entropy formalism, in: Ed. Levine, R. D., Tribus, M., Where do we stand...?, MIT, Cambridge, 1979.

[8] Muschik, W., Kaufmann, M., Quantum-Thermodynamical Description of Discrete NonEquilibrium Systems, J. Non-Equilib. Thermodyn., 19 (1994), 76-94. 
[9] Mori, H., A Continued Fraction Representation of the Time-Correlation Functions, Progr. Theor. Phys., 34, (1965), 399-416.

[10] Fick, E., Sauermann, G., Quantum Statistics of Dynamic Processes, Springer, Heidelberg, 1990.

[11] Kawasaki, K., Gunton, J. D., Theory of nonlinear transport processes: Nonlinear shear viscosity and normal stress effects, Phys. Rev. A, 8 (1973), 2048-2064.

[12] Robertson, B., Equations of motion in nonequilibrium statistical mechanics, Phys. Rev., 144 (1966), 151.

[13] Schirrmeister, D., Unterschiedliche mikroskopische Dynamiken zur Quantenthermodynamik unter Berücksichtigung explizit zeitabhängiger thermodynamischer Zustände, Diplomarbeit, Institut für Theoretische Physik, Technische Universtität Berlin, 1994.

[14] Muschik, W., Empirical foundation and axiomatic treatment of non-equilibrium temperature, Arch. Rat. Mech. Anal., 66 (1977), 379-401.

[15] Muschik, W., Brunk, G., A concept of non-equilibrium temperature, Int. J. Engng. Sci., 15 (1977), 377.

[16] Muschik, W., Contact temperature, a dynamical analogue of the themostatic temperature, in: Continuum Models of Discrete Systems 4, Eds. Brulin, O., Hsieh, R. K. T., NorthHolland, Amsterdam, 1981, 511.

[17] Muschik, W., Aspects of Non-Equilibrium Thermodynamics, 6 Lectures on Fundamentals and Methods, World Scientific, Singapore, 1990, Sect. 4.1.

[18] Muschik, W., Fang, J., Statistical foundation of nonequilibrium contact quantities bridging phenomenological and statistical nonequilibrium thermodynamics, Acta Phys. Hung., 66 (1989), 39-57.

Paper received: 1999-8-5

Paper accepted: 2000-2-2
A. Kato
M. Kaufmann
W. Muschik ${ }^{2)}$
D. Schirrmeister
Institut für Theoretische Physik
Technische Universität Berlin PN 7-1
Hardenbergstraße 36
10623 Berlin
Germany

${ }^{2)}$ Corresponding author: muschik@ $@$ physik.tu.-berlin.de 